\title{
ESTUDO ESTRUTURAL E TERMOFÍSICO DE UMA LIGA DE NiTi SUBMETIDA A DIFERENTES TRATAMENTOS DE SOLUBILIZAÇÃO*
}

\author{
Patricia Freitas Rodrigues ${ }^{1}$ \\ Francisco Manuel Braz Fernandes ${ }^{2}$ \\ Andersan dos Santos Paula \\ Shimeni Baptista Ribeiro ${ }^{4}$ \\ Edir Neves Teixeira ${ }^{5}$ \\ Saulo Brinco Diniz
}

\section{Resumo}

Este trabalho visou compreender as características microestruturais de uma liga de $\mathrm{NiTi}$ rica em Ni na forma de discos com $10 \mathrm{~mm}$ de diâmetro forjados a quente, que foram submetidos a um tratamento térmico de solubilização com distintas temperaturas e tempos de encharque. Foram testadas as seguintes condições: $800^{\circ} \mathrm{C} / 10,800^{\circ} \mathrm{C} / 120 \mathrm{~min}, 850^{\circ} \mathrm{C} / 120 \mathrm{~min}$ e $950^{\circ} \mathrm{C} / 120 \mathrm{~min}$. Foi observado através dos ensaios de DSC e DRX com radiação de synchrotron que o tratamento de solubilização com $950^{\circ} \mathrm{C} / 120 \mathrm{~min}$ é o mais adequado para o tratamento de solubilização prévia ao processamento termomecânico a ser aplicado nesta liga afim de produzir no final deste processamento fios ortodônticos.

Palavras-chave: Ligas com memória de forma; NiTi; Tratamento Térmico de Solubilização.

\section{STRUCTURAL AND THERMOPHYSIC STUDY OF A NITI ALLOY SUBMITTED TO DISTINCT SOLUTION HEAT TREATMENTS}

\section{Abstract}

This work aimed to understand the microstructural characteristics of a NiTi alloy rich in Ni samples with $10 \mathrm{~mm}$ diameter, which were subjected to solution heat treatment by different temperatures and soaking times. The conditions tested were: $800^{\circ} \mathrm{C} / 10 \mathrm{~min}, 800^{\circ} \mathrm{C} / 120 \mathrm{~min}, 850^{\circ} \mathrm{C} / 120 \mathrm{~min}$ and $950^{\circ} \mathrm{C} / 120 \mathrm{~min}$. It was observed by the DSC and XRD (synchrotron radiation) tests that the solution heat treatment with $950^{\circ} \mathrm{C} / 120 \mathrm{~min}$ is most suitable for the solution heat treatment prior to the thermomechanical processing to be applied in this alloy in order to produce orthodontic wires.

Keywords: Shape memory alloys; NiTi; Solution Heat Treatment.

1 Bacharel em Química Industrial, M.Sc., Doutoranda em Ciência e Engenharia de Materiais, Cenimat/i3N, DCM/FCT/UNL, Caparica, Portugal; e Bolsista Capes.

* Bolsista, CAPES - Foundation Ministry of Education of Brazil, Brasília - DF - Brazil

2 Engo Mecânico, M.Sc., D. Sc., Professor Associado, Cenimat/i3N, DCM/FCT/UNL, Caparica, Portugal.

3 Enga Metalurgista, M.Sc., D.Sc., Professora Adjunta, IME, Rio de Janeiro, RJ, Brasil.

4 Eng ${ }^{a}$ Metalurgista, M.Sc., D.Sc., Professora, UniFOA, Volta Redonda, RJ, Brasil.

5 Engo Metalurgista, M.Sc., Professor, UniFOA, Volta Redonda, RJ, Brasil

6 Eng ${ }^{\circ}$ Metalurgista, M.Sc., Doutorando em Ciência dos Materiais, IME, Rio de Janeiro, RJ, Brasil. 


\section{INTRODUÇÃO}

Ligas de NiTi, despertam um grande interesse de estudo por suas características distintas, tais como: o efeito de memória de forma e a superelasticidade [1]. Essas propriedades dependem da ocorrência da transformação martensítica termoelástica, para a apresentarem a capacidade de retomar a uma forma ou dimensão previamente definida quando são submetidas a tratamentos termomecânicos apropriados [2].

As ligas com composição próxima a equiatômica apresentam duas fases: austenita (B2 - estável a mais altas temperaturas) e martensita (B19' - estável em temperaturas mais baixas). Em algumas situações uma fase intermediaria, fase $R$, pode aparecer. As temperaturas de transformação nessas ligas são dependentes da composição química, relação $\mathrm{Ni} / \mathrm{Ti}$ em sua matriz B2, e nível de tensão residual. Para algumas aplicações o comportamento superelástico é muito importante devido a transformação induzida por tensão B2 $\leftrightarrow$ B19'.

O processamento neste tipo de liga é complexo, desde a sua elaboração, uma vez que a composição química e a presença de impurezas ( $\mathrm{C}$ e/ou $\mathrm{O})$ podem modificar drasticamente as características do material [3-5]. Um dos desafios no processamento deste tipo de liga é o controle da composição da matriz metálica (Ni/Ti) e presença de impurezas, que podem dar origem a carbetos $(\mathrm{TiC})$ e óxidos $\left(\mathrm{Ti}_{4} \mathrm{Nl}_{2} \mathrm{O}\right.$ e $\left.\mathrm{Ti}_{2} \mathrm{O}\right)[1,6-8]$. A presença de carbono e oxigênio não alteram diretamente a temperatura de transformação de fase. No entanto, os elementos irão se combinar com o Ti para formar compostos estáveis, assim a matriz se apresentará rica em $\mathrm{Ni}$ e consequentemente promove uma redução na temperatura de transformação de fase $[3,4,9]$.

Compreender o comportamento desses materiais durante seu processamento de fabricação é muito importante, especialmente durante as etapas de deformação a quente $[8,9]$. Esta etapa de processamento termomecânico, para além de ser responsável por prover significativas reduções em determinada dimensão, é também responsável por acelerar o processo de homogeneização microestrutural em função da combinação de ação térmica e mecânica que ativam fortemente processo difusionais. Mas por outro lado, também pode vir a introduzir níveis de tensões residuais no material. A sua capacidade de deformação a quente pode ser reduzida por níveis elevados de inclusões oriundas da etapa de fusão/elaboração da liga [10]. Torna-se desejável que este material apresente uma condição homogênea para o processamento, por isso geralmente se propõem uma solubilização para garantir uma composição livre de precipitados e tensões residuais baixas capazes de afetar as etapas de processamento, para garantir a estabilidade de B2 a temperatura ambiente. Para isso busca-se um aquecimento controlado a uma dada temperatura durante um tempo suficiente para resultar em material monofásico durante o encharque que por meio de um resfriamento impeça a formação de precipitados e outras fases [11]. Geralmente esses tratamentos térmicos são superiores a $800^{\circ} \mathrm{C}$ para a composição próxima a equiatômica e rica em Ni. Como a liga em estudo é uma liga com $50,8 \%$ at $\mathrm{Ni}$, foram observadas as temperaturas de 800,850 e $950^{\circ} \mathrm{C}$ neste trabalho.

$\mathrm{Na}$ Figura 1, observa-se a gama de composição e as temperaturas selecionadas para este estudo. 


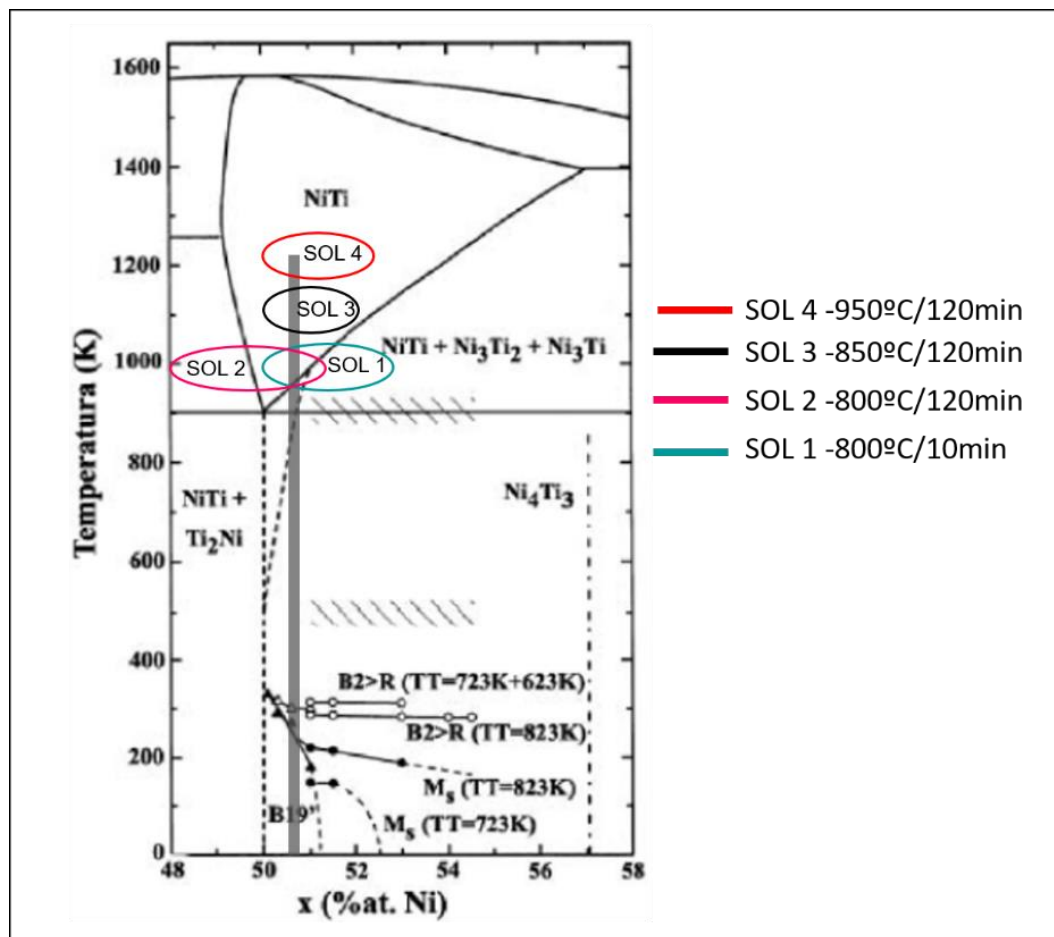

Figura 1. Diagrama parcial de equilíbrio do sistema NiTi [adaptado de 13].

Objetivo deste trabalho é compreender e identificar a melhor condição para tratamento térmico de solubilização em uma liga de $\mathrm{NiTi}$ rica em $\mathrm{Ni}(50.8 \% a \mathrm{Ni})$. Esta solubilização busca obter uma amostra majoritariamente austenítica (B2) a temperatura ambiente (com $A_{f}$ inferior a $25^{\circ} \mathrm{C}$ ). Para isso o tratamento precisa promover completa dissolução de precipitados associados ao $\mathrm{Ni}$ e ao $\mathrm{Ti}$ e ainda promover a remoção de tensões existentes como vestígio da primeira etapa do processamento de forjamento rotativo a quente.

\section{MATERIAIS E MÉTODOS}

O material estudado é correspondente a primeira etapa de forjamento a quente (1F1q - Figura 2), barras com diâmetro de $10 \mathrm{~mm}$ e uma composição de $50,8 \%$ at $\mathrm{Ni}$, fornecida pelo professor e pesquisador Dr. Jorge Otubo do Instituto Tecnológica da Aeronáutica (ITA), Brasil.

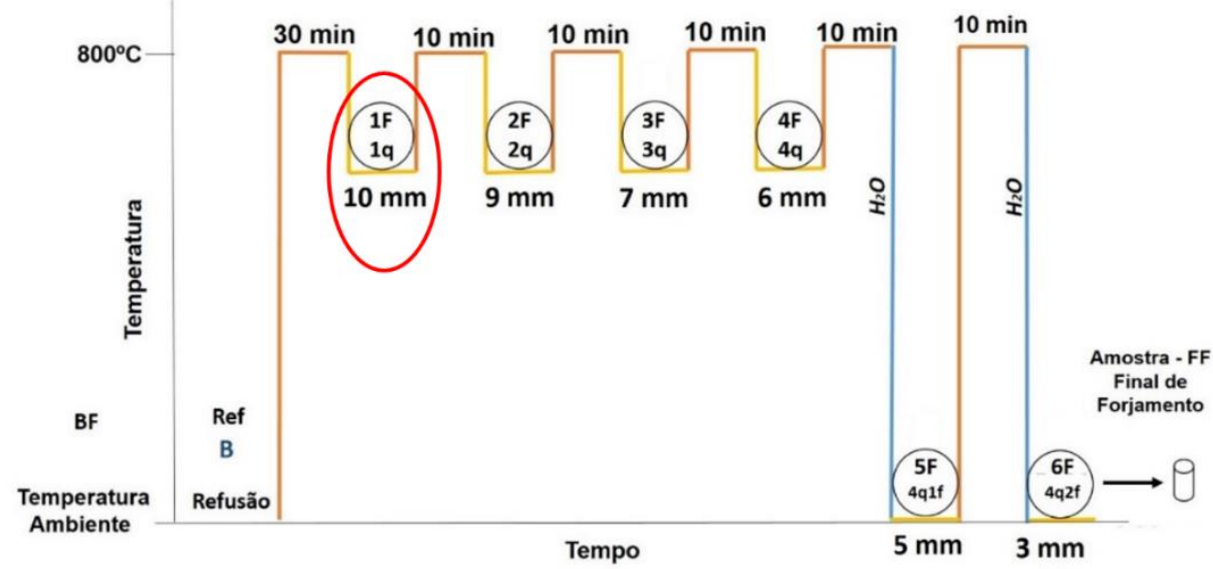

Figura 2. Processamento termomecânico do lingote de uma liga NiTi rica em Ni, com etapas de forjamento rotativo, temperatura/tempos de encharque dos tratamentos térmicos intermediários e diâmetros das amostras. 
Para este estudo, cinco amostras foram cortadas da condição 1F1Q (Figura 2), por um processo de corte abrasivo em uma máquina de corte de precisão com o auxílio de disco diamantado com resfriamento com água. As amostras foram solubilizadas em diferentes condições em forno mufla e depois resfriadas em água até temperatura ambiente. As condições de tratamento térmico são apresentadas na Tabela 1.

As medidas de temperatura de transformação de fase foram realizadas por um equipamento Netzsch modelo F401, Proteus, com forno de baixa temperatura $\left(-150^{\circ} \mathrm{C}\right.$ a $600^{\circ} \mathrm{C}$ ), instalado no Centro de Investigação de Materiais (CENIMAT), na Faculdade de Ciências e Tecnologia da Universidade Nova de Lisboa.

Primeiro foi verificado as temperaturas na amostra tal como retirada do processamento realizado (1F1Q) e depois foi realizada uma simulação do tratamento térmico aplicado no processamento em estudo $\left(800^{\circ} \mathrm{C} / 10 \mathrm{~min}\right)$ visando a condição de entrada na forja no segundo passe de forjamento (2F2Q), no intuito de compreender o comportamento termofísico desta etapa. Após análise desta etapa foram propostos outros tratamentos térmicos. O tempo e temperatura dos tratamentos térmicos foram baseados em estudos anteriores [14,15]. As amostras foram pesadas sempre respeitando a massa de aproximadamente $10 \mathrm{mg}$. Para este ensaio as amostras foram submetidas a ciclos térmicos de $150^{\circ} \mathrm{C}$ até $-150^{\circ} \mathrm{C}$ com taxa de aquecimento e resfriamento de $10^{\circ} \mathrm{C} / \mathrm{min}$.

A partir das curvas de DSC, as temperaturas de início e fim das transformações de fase foram determinadas pela interseção de uma linha de base tangente a um pico. Os valores de temperatura de transformação de fase final da transformação B19' $\rightarrow$ $\mathrm{B} 2$, foram comparados com a temperatura ambiente $\left(25^{\circ} \mathrm{C}\right)$.

As amostras para DRX foram extraídas de maneira semelhante as amostras para ensaio de DSC. Após o corte, essas foram submetidas a um lixamento de uma de suas faces, com auxílio de uma lixadeira rotativa manual com lixas de carbeto de silício até 4000 mesh. Posteriormente a etapa de lixamento, as amostras foram imersas em uma solução de $45 \mathrm{ml}$ de $\mathrm{HNO}_{3}, 45 \mathrm{ml}$ de $\mathrm{H}_{2} \mathrm{O}$ e $10 \mathrm{ml} \mathrm{HF}$, por aproximadamente 60 s. Esta decapagem ácida foi realizada com intuito de remover a camada deformada proveniente da etapa de corte e lixamento.

As fases presentes a temperatura ambiente, foram analisadas com o auxílio da análise da difração de raios-X usando radiação de synchrotron (linha P07 High-Energy Materials Science / HEMS, Petra III, DESY). Este experimento foi realizado em modo de transmissão em amostras com $10 \mathrm{~mm}$ de diâmetro, com comprimento de onda de $0,1426 \AA$ (87keV), feixe de 200x200 $\mu \mathrm{m}^{2}$, por um detector 2D Perkin Elmer posicionado a uma distância de $1390 \mathrm{~mm}$ da amostra. As imagens foram tratadas usando o programa Fit2D para calcular os padrões DRX, por integração para Phi (azimute) de $0^{\circ}$ a 360․ A identificação dos picos foi baseada no banco de dados ICDD.

Tabela 1. Nome e Tratamento Térmico.

\begin{tabular}{|c|c|c|}
\hline Nome & $\begin{array}{c}\text { Temperatura de Tratamento } \\
\text { (ํ) }\end{array}$ & $\begin{array}{c}\text { Tempo de Tratamento } \\
\text { (min.) }\end{array}$ \\
\hline 1F1Q & - & - \\
\hline SOL 1 & 800 & 10 \\
\hline SOL 2 & 800 & 120 \\
\hline SOL 3 & 850 & 120 \\
\hline SOL 4 & 950 & 120 \\
\hline
\end{tabular}




\section{RESULTADOS E DISCUSSÃO}

Os efeitos do tratamento térmico de solubilização sobre o comportamento da temperatura de transformação de fase são apresentados na Figura 2.

Os pontos $A_{s}$ e $A_{f}$ são temperaturas de início e fim da transformação austenítica, os pontos $M_{s}$ e $M_{f}$ são as temperaturas de início e final da transformação martensítica.

A amostra explorada neste estudo, foi processada a quente uma vez, ou seja, somente com um único e primeiro passe de forjamento (conforme Figura 2).

Nos ensaios de DSC, pode-se observar para algumas situações a existência de faixas de temperaturas sobrepostas tanto no resfriamento quanto no aquecimento, com indicação de possível presença de fase R. Esta fase pode aparecer antes da transformação martensítica sob algumas condições de tratamentos, tais como, tratamento térmico e ainda sob uma certa condição de trabalho a quente.

Estudos diversos apresentam alterações significativas das características de transformação para tratamentos térmicos acima de $500^{\circ} \mathrm{C}[13,16]$. Quando esta temperatura é superior a temperatura de recristalização, vários mecanismos podem estar envolvidos tais como: crescimento de grãos, precipitação, alteração da textura, etc [16].

$\mathrm{Na}$ Figura 3, pode-se observar que aumentando o tempo de encharque, aumenta a temperatura $A_{f}$. No entanto, o comportamento inverso é observado com o aumento da temperatura de tratamento para o mesmo tempo de encharque, exceto pela amostra $950^{\circ} \mathrm{C} / 120 \mathrm{~min}$. Esta amostra apresenta estabilidade de B2 a temperatura ambiente

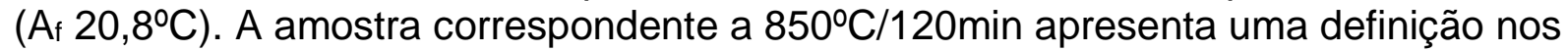
picos tanto no resfriamento quanto no aquecimento, isso pode estar relacionado a uma redução de defeitos estruturais e ainda uma redução de tensões internas 


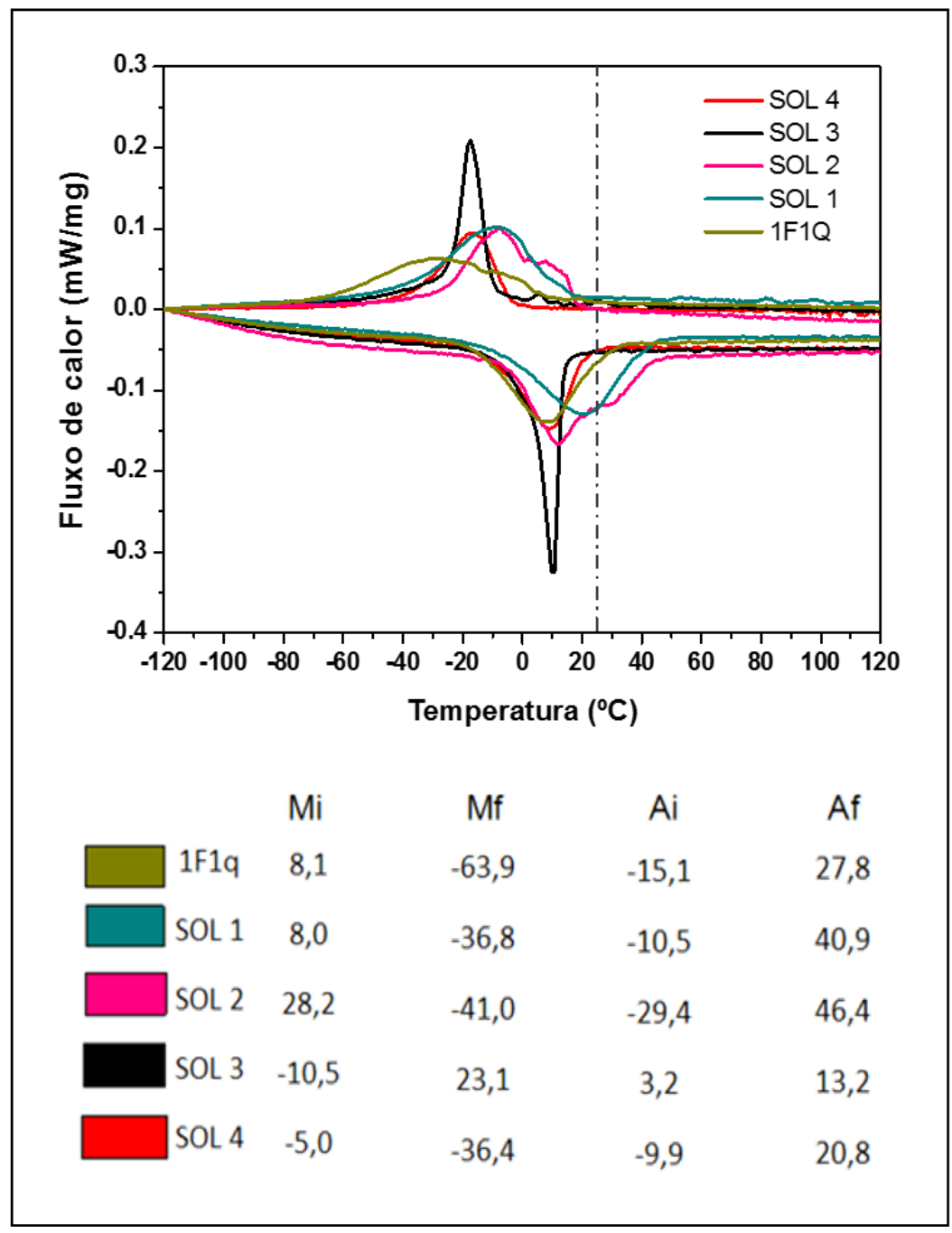

Figura 3. Temperaturas de Transformação de fases. - a) Curvas de DSC com destaque da temperatura ambiente $\left(25^{\circ} \mathrm{C}\right)$ b) Temperaturas de transformação de fases inicias $\left(A_{s}\right.$ e $\left.M_{s}\right)$ e finais $\left(A_{f}\right.$ e $\left.\mathrm{M}_{\mathrm{f}}\right)$.

A amostra (1F1q) apresenta um pico de transformação mais alargado não muito bem definido, esta característica pode estar associada ao encruamento da fase B2. Essa amostra ainda apresenta indicativos de transformação com duas etapas (B2↔R↔B19'), possivelmente associado a regiões com heterogeneidade composicional devido a precipitação da fase metaestável $\mathrm{Ni}_{4} \mathrm{Ti}_{3}$ [1].

Para os ensaios de DRX pode-se observar na Figura 4, que o menor tempo na mesma temperatura de tratamento térmico (SOL $1-800^{\circ} \mathrm{C} / 10$ e SOL 2 - 800ํㄷ120min) apresenta uma diferença na intensidade difratada. 


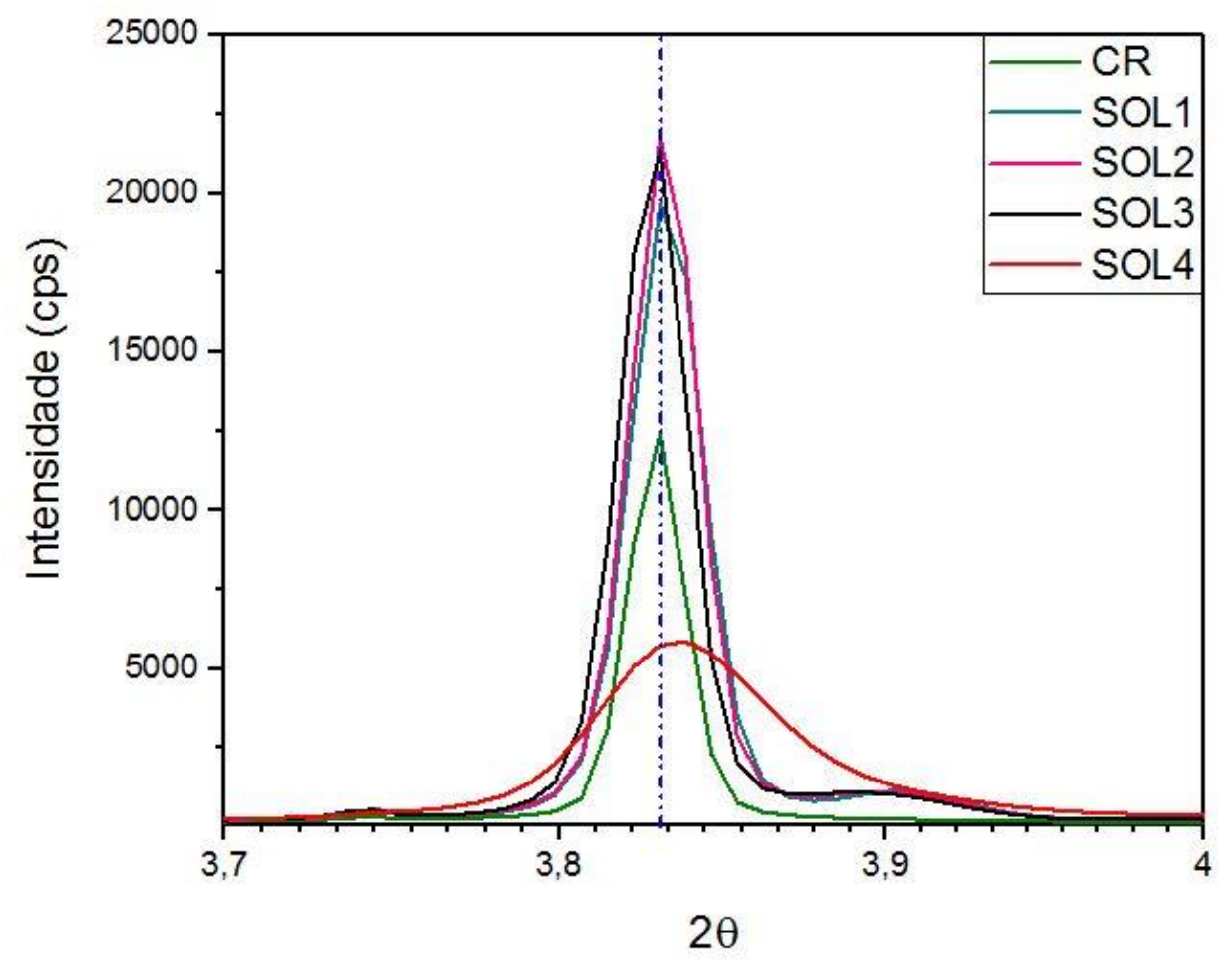

Figura 4. Difratograma das amostras para o pico B2 (110), em função da radiação com comprimento de onda comprimento de onda de 0,1426 ̊.

Observa-se uma assimetria nos picos isso possivelmente associado a sobreposição de picos entre as fases B2 (110) e R ((20-2) e (220)).

Para as amostras correspondentes ao mesmo tempo e aumento de temperatura de encharque (Figura 5), observa-se que a amostra com $800^{\circ} \mathrm{C} / 120 \mathrm{~min}$ (SOL2) apresenta um aumento de intensidade para o pico B2 (110), com possível sobreposição de Fase R. Para as amostras com tratamento de $800^{\circ} \mathrm{C} / 120 \mathrm{~min}$ (SOL2) e $850^{\circ} \mathrm{C} / 120 \mathrm{~min}$ (SOL3) pode-se observar uma boa definição para pico B2 (110), a identificação do precipitado $\mathrm{Ni}_{4} \mathrm{Ti}_{3}$ e ainda B19' (021). A amostra $950^{\circ} \mathrm{C} / 120 \mathrm{~min}$ apresenta uma menor intensidade para o pico B2 (110) e não foi possível identificar o precipitado $\mathrm{Ni}_{4} \mathrm{Ti}_{3}$ nesta condição de tratamento térmico.

Isso pode estar associado a dissolução dos precipitados presentes nesta amostra, quanto maior a temperatura de encharque menor a dimensão e fração volumétrica dos precipitados que se faziam presentes. Isto ainda irá influenciar na redução das temperaturas de transformação de fase e estabilizará a fase B2 a temperatura ambiente, como discutido anteriormente. 

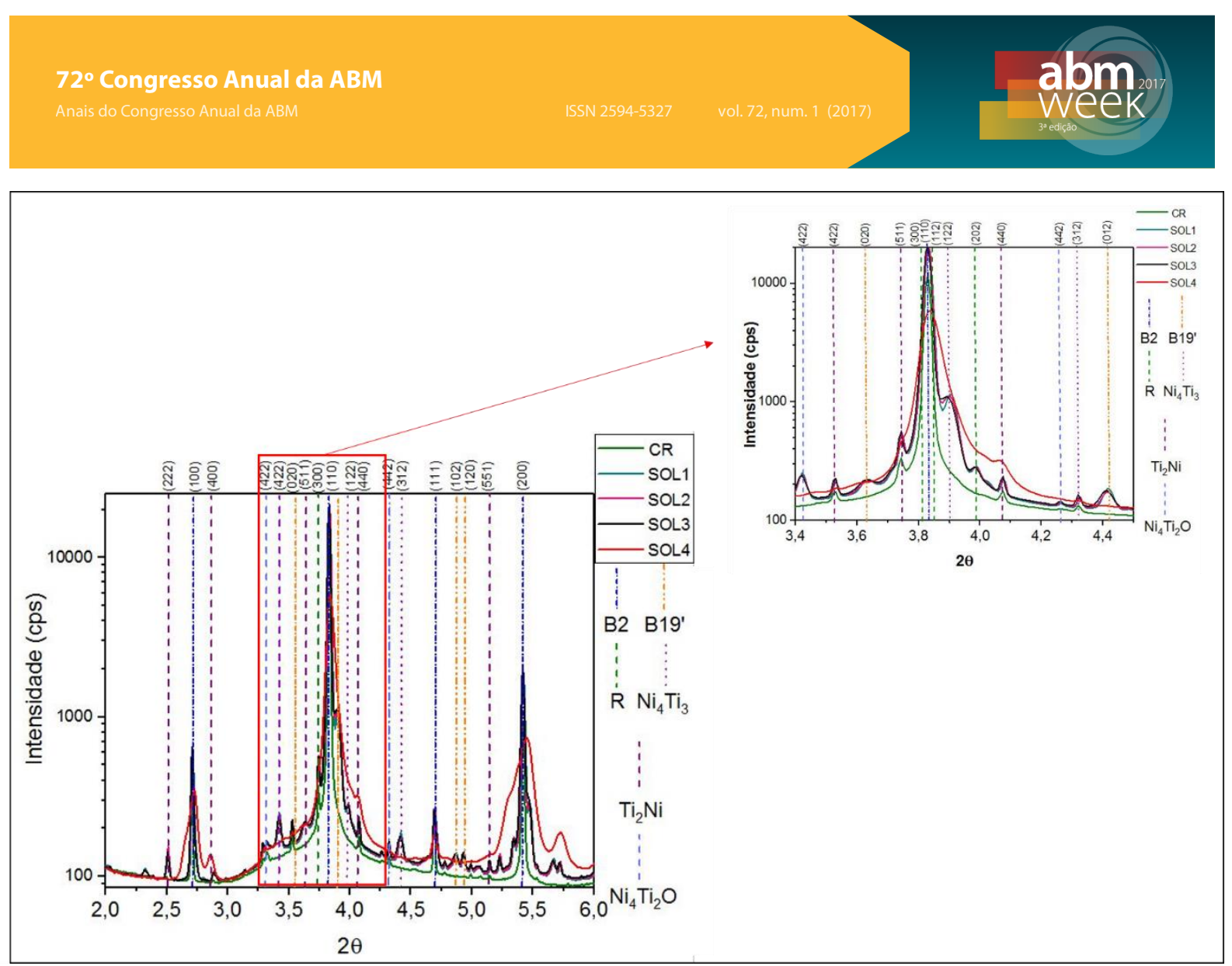

Figura 5. Difratograma comparativo para as amostras $1 \mathrm{~F} 1 \mathrm{q}(\mathrm{CR})$, SOL1 $\left(800^{\circ} \mathrm{C} / 10 \mathrm{~min}\right)$, SOL2 $\left(800^{\circ} \mathrm{C} / 120 \mathrm{~min}\right)$, SOL3 $\left(850^{\circ} \mathrm{C} / 120 \mathrm{~min}\right)$ e SOL4 $\left(950^{\circ} \mathrm{C} / 120 \mathrm{~min}\right)$, ensaios realizados à temperatura ambiente.

Para continuidade de estudos do processamento termomecânico na liga de estudo, com intuito de otimizar os tratamentos térmicos de solubilização para ser aplicado previamente a este, análises de DRX convencional, DRX in-situ a alta temperatura e ainda dilatometria e estudo detalhado relacionado ao DRX - synchrotron estão sendo realizados. Com isso será possível identificar e compreender as fases existentes e suas relações com as temperaturas de tratamento térmico e sua influência à temperatura ambiente.

\section{CONCLUSÃO}

A análise dos resultados DSC e DRXS do tratamento de solubilização na amostra correspondente a primeira etapa de forjamento rotativo a quente resultou em:

- Para as amostras com tratamento térmico a $800^{\circ} \mathrm{C}$ (10 e $120 \mathrm{~min}$ ), a temperatura de $A_{f}$ é superior à temperatura ambiente, apresentando por isso uma mistura de fases à temperatura ambiente.

- A temperatura de $A_{f}$ para as amostras com tratamento de 850 e 950ㄷ (120 min) apresentaram valores inferiores a temperatura ambiente, estabilizando assim a fase austenita (B2) a temperatura de interesse $\left(25^{\circ} \mathrm{C}\right)$.

- Tratamento térmico de $950^{\circ} \mathrm{C} / 120 \mathrm{~min}$ foi capaz de promover a dissolução dos precipitados oriundos do processamento, garantindo assim uma matriz homogênea e livre de tensões.

- Foi possível identificar transformações de fase em duas etapas (B2 $R \leftrightarrow B 19$ ')

- Foi possível identificar uma heterogeneidade no material estudado, isso é relacionado ao processamento de fusão realizado na amostra. 
- Apesar de identificar uma heterogeneidade nas amostras analisadas, para este caso específico pode-se assegurar a solubilização prévia de $950^{\circ} \mathrm{C} / 120 \mathrm{~min}$ como condição de tratamento térmico para este tipo de liga.

\section{Agradecimentos}

Os autores agradecem ao professor J. Otubo por doar os materiais de partida, que foi elaborado no Instituto de Tecnologia Aeronáutica (São José dos Campos, SP - Brasil). P.F.R e F.M.B.F reconhecem o financiamento do CENIMAT / i3N pelo COMPETE 2020, através da FCT, no âmbito do projeto UID/CTM/50025/2013.

A autora P.F.R. agradece a bolsa de doutorado subsidiada pela CAPES vinculada ao programa Ciências Sem Fronteiras - BEX 11943-13-0.

A.S.P. agracede a bolsa de produtividade à pesquisa (PQ-2) do CNPq (Processo 307798/2015-1).

\section{REFERÊNCIAS}

1 OTSUKA, K., REN, X. Physical Metallurgy of Ti-Ni-based Shape Memory Alloys. Progress in Materials Science, 50, 2005.

2 MASSALSKI, T.B et al. Binary alloy phase diagrams. Materials Park, $\mathrm{OH}$ : ASM

3 CHU, C. L., WU, S. K., and YEN, Y. C. "Oxidation behavior of equiatomic TiNi alloy in high temperature air environment," Mater. Sci. Eng. A, 216, 193-200, 1996.

4 FRENZEL, J., ZHANG, Z., SOMSEN, C., NEUKING, K., and EGGELER, G. "Influence of carbon on martensitic phase transformations in Niti shape memory alloys," Acta Mater., 55, 1331-1341, 2007.

5 OTUBO, J., RIGO, O. D., COELHO, A. A., NETO, C. M., and MEI, P. R. "The influence of carbon and oxygen content on the martensitic transformation temperatures and enthalpies of NiTi shape memory alloy," Mater. Sci. Eng. A 481-482, 639-642, 2008.

FUNAKUBO, H. Shape Memory Alloys (Science Publishers, New York, Gordon and Breach), Vol. 1, 1987.

$7 \mathrm{KHAMEI}, \mathrm{A}$. and DEHGHANI, K. "Microstructural evolution during the hot deformation of Ti-55Ni (at. pct) intermetallic alloy," Metall. Mater. Trans. A 41, 2595-2605, 2010.

8 DEHGHANI, K. and KHAMEI, A. A. "Modeling the hot-deformation behavior of Ni60 wt\%Ti40 wt\% intermetallic alloy," J. Alloys Compd. 490, 377-381, 2010.

9 FRENZEL, J., George, E. P., Dlouhy, A., Somsen, Ch., Wagner, M. F.-X., and Eggeler, G. "Influence of Ni on martensitic phase transformations in NiTi shape memory alloys," Acta Mater. 58, 3444-3458, 2010.

10 OTUBO, J., RIGO, O. D., MOURA NETO, C., and MEI, P. R. "The effects of vacuum induction melting and electron beam melting techniques on the purity of NiTi shape memory alloys," Mater. Sci. Eng. 438-440, 679-682, 2006.

11 LIU, Y; VAN HUMBEECK, J. J. Physique, v.5, pp.519, 1997.

12 COSTA E SILVA A.L., MEI P. R. Aços e Ligas Especiais - Sumaré-SP: Eletrometal 1988.

13 SABURI, T; NENNO, S. Proc Int Conf Solid-Solid phase Transformations.Warrendale, 1981.

14 TEXEIRA, E.; Otimização Das Condições Dos Tratamentos Térmicos De Solubilização e Envelhecimento Em Arame Forjado De Liga De NiTi Rica Em Ni Para Aplicações Superelásticas, Dissertação de Mestrado, Universidade Federal Fluminense-UFF, Brazil, 2013.

15 RIBEIRO, S. B., Recuperação e recristalização de liga NiTi deformada por compressão e tratada termicamente no intervalo entre 150 e $500^{\circ} \mathrm{C}$, Tese de Doutorado UFF/PPGEM 2015. 
16 YEOM, J.-T., KIM, J. H., HONG, J.-K., KIM, S.W., PARK, C.-H., NAM, T. H., and LEE, K.-Y. "Hot forging design of as-cast NiTi shape memory alloy," Mater. Res. Bull. 58, 234-238, 2014. 